\title{
Effectiveness of bronchoscopic lung volume reduction using unilateral endobronchial valve: a systematic review and meta-analysis
}

This article was published in the following Dove Press journal:

International Journal of COPD

3I March 2015

Number of times this article has been viewed

Miyoung Choi'

Worl Suk Lee'

Min Lee'

Kyeongman Jeon ${ }^{2}$

Seungsoo Sheen ${ }^{3}$

Sanghoon Jheon ${ }^{4}$

Young Sam Kim ${ }^{5}$

'National Evidence-Based Healthcare Collaborating Agency, Seoul, Republic of Korea; ${ }^{2}$ Division of Pulmonary and Critical Care Medicine, Department of Medicine, Samsung Medical Center, Sungkyunkwan University School of Medicine, Seoul, Republic of Korea; ${ }^{3}$ Department of Pulmonary and Critical Care Medicine, Ajou University School of Medicine, Suwon, Republic of Korea; ${ }^{4}$ Department of Thoracic and Cardiovascular Surgery, Seoul National University Bundang Hospital, Seoul National University College of Medicine, Seoul, Republic of Korea; ${ }^{5}$ Department of Internal Medicine, Severance Hospital, Yonsei University College of Medicine, Seoul, Republic of Korea
Correspondence: Young Sam Kim Department of Internal Medicine, Severance Hospital, Yonsei University College of Medicine, 50-I Yonsei-Ro, Seodaemun Gu, Seoul, Republic of Korea Tel +82 22228 | $97 \mid$

Fax +82 23936884

Email ysamkim@yuhs.ac
Background: Bronchoscopic lung volume reduction (BLVR) can be suggested as an alternative for surgical lung volume reduction surgery for severe emphysema patients. This article intends to evaluate by systematic review the safety and effectiveness of BLVR using a one-way endobronchial valve.

Methods: A systematic search of electronic databases, including MEDLINE, EMBASE, and the Cochrane Library, as well as eight domestic databases up to December 2013, was performed. Two reviewers independently screened all references according to selection criteria. The Scottish Intercollegiate Guidelines Network criterion was used to assess quality of literature. Data from randomized controlled trials were combined and meta-analysis was performed.

Results: This review included 15 studies. Forced expiratory volume in 1 second $\left(\mathrm{FEV}_{1}\right)$ improved in the intervention group compared with the control group (mean difference $[\mathrm{MD}]=6.71,95 \%$ confidence interval [CI]: 3.31-10.11). Six-minute walking distance (MD=15.66, 95\% CI: 1.69-29.64) and cycle workload ( $\mathrm{MD}=4.43,95 \%$ CI: $1.80-7.07)$ also improved. In addition, St George's Respiratory Questionnaire score decreased (MD=4.29, 95\% CI: -6.87 to -1.71 ) in the intervention group. In a subgroup analysis of patients with complete fissure, the $\mathrm{FEV}_{1}$ change from baseline was higher in the BLVR group than in the control group for both 6 months $(\mathrm{MD}=15.28, P<0.001)$ and 12 months $(\mathrm{MD}=17.65, P<0.001)$, whereas for patients with incomplete fissure, $\mathrm{FEV}_{1}$ and 6-minute walking distance showed no change. One-year follow-up randomized controlled trials reported deaths, although the cause of death was not related to BLVR. Respiratory failure and pneumothorax incidence rates were relatively higher in the BLVR group, but the difference was not significant.

Conclusion: BLVR may be an effective and safe procedure for the treatment of severe COPD patients with emphysema, based on existing studies.

Keywords: endobronchial valve, bronchoscopic valve lung volume reduction, systematic review, meta-analysis

\section{Introduction}

Chronic obstructive pulmonary disease (COPD) is a respiratory disease characterized by irreversible abnormal lung inflammation and shows progressive airflow limitation. ${ }^{1}$ It is the fifth leading cause of death in high-income countries ${ }^{2}$ and is one of the major public health problems across the world. According to the Global initiative for chronic Obstructive Lung Disease (GOLD), it is predicted that COPD will be the third cause of death in $2020 .^{3}$ To reduce hyperinflation, lung volume reduction surgery for severe emphysema has been performed for over 30 years. Surgery-related complications and mortality are the main concerns with this form of treatment. ${ }^{4}$ 
Bronchoscopic lung volume reduction (BLVR) was introduced to maintain the effect of lung volume reduction and also reduce surgery-related complications and mortality. ${ }^{5}$ Many studies of BLVR have been published, with the majority of study designs being case series. Therefore, randomized controlled trials (RCTs) have been recommended for evidence enhancement. ${ }^{6}$ Recently, the US and Europe have reported results of multicenter RCTs using unilateral bronchial valves for BLVR, but meta-analysis is not yet published. ${ }^{7,8}$ This study is a systematic review to evaluate the effectiveness and safety of BLVR using unilateral bronchial valves in COPD with emphysema.

\section{Materials and methods}

\section{Search strategy}

A literature search was performed using eight domestic research databases, including KoreaMed, and core databases such as MEDLINE, EMBASE, and the Cochrane Library up to December 2013. Key question and patient intervention comparators and outcomes were defined under the advice of expert groups. The presearch included 148 article abstracts. Extensive searches of databases using the terms "endoscopic lung volume reduction", "bronchoscopic lung volume reduction", "endobronchial valve", and "BLVR" were performed. Terms were related to the intervention and modified according to each database's index term, such as Medical Subject Heading (MeSH) and EMTREE.

\section{Inclusion/exclusion criteria}

Studies that met the following criteria were included: 1) study population is people with COPD with severe emphysema; 2) intervention is BLVR using a unilateral bronchial valve; 3) study design is an RCT, a cohort study, or a case series; 4) reported one of the predetermined outcomes; and 5) published in Korean or English.

Studies on animal trial or preclinical studies and nonoriginal articles such as reviews, editorials, letters, and comments were excluded. Articles not published in either Korean or English and studies with duplicate subjects (study using the same outcome indicators published in duplicate) were also excluded.

\section{Types of outcome measures}

Primary outcome was lung function at forced expiratory volume in 1 second $\left(\mathrm{FEV}_{1}\right)$. Secondary outcomes were 6-minute walk distance (6MWD), cycle workload (Watt), quality of life: St George's Respiratory Questionnaire
(SGRQ) score, dyspnea scale: modified Medical Research Council score, and safety issues: major complications (death, pneumothorax, massive hemoptysis) and minor complications (pneumonia, minor hemoptysis, COPD exacerbation).

\section{Data collection and analysis Selection of studies and quality assessment}

Each stage, from the literature search to the application of selection criteria and data extraction, was independently undertaken by two researchers. Study selection was carried out by predefined inclusion/exclusion criteria. An expert group consisting of respiratory medicine and evidence-based medicine specialists and thoracic surgery specialists in COPD guided each stage. Quality assessment of selected studies was carried out using tools of the Scottish Intercollegiate Guidelines Network. $^{9}$

\section{Data extraction and management}

According to a preagreed data extraction format, two investigators independently extracted data for review. Continuous variables such as mean change from baseline, median range, and standard deviation were converted according to the formula in the Cochrane Handbookfor Systematic Reviews of Interventions. ${ }^{10}$ The extracted data syntheses were performed both quantitatively and qualitatively.

\section{Measurement and treatment effect}

The mean difference (MD) and 95\% confidence interval (CI) from each individual RCT were calculated, and metaanalysis using a fixed effect model was performed. ${ }^{11}$ Two $\mathrm{RCTs}^{7,8}$ were included in the meta-analysis. One set of data from an $\mathrm{RCT}^{12}$ was not included, because the comparator and follow-up period were different. Statistical analysis was performed using Cochrane RevMan version 5.2 and Comprehensive Meta Analysis software.

\section{Results \\ Study characteristics}

A total of 1,016 studies were retrieved from the database. After exclusion of duplicates, 678 studies remained. Finally, 15 studies were selected according to the selection and exclusion criteria (Figure 1): three RCTs, ${ }^{7,8,12}$ one cohort study, ${ }^{13}$ and eleven case studies. ${ }^{14-24}$ All studies used a duckbill-type endobronchial valve. Six studies used a $\mathrm{Zephyr}^{\circledR}$ valve and eight studies used an Emphasys ${ }^{\circledR}$ valve (Table 1). 


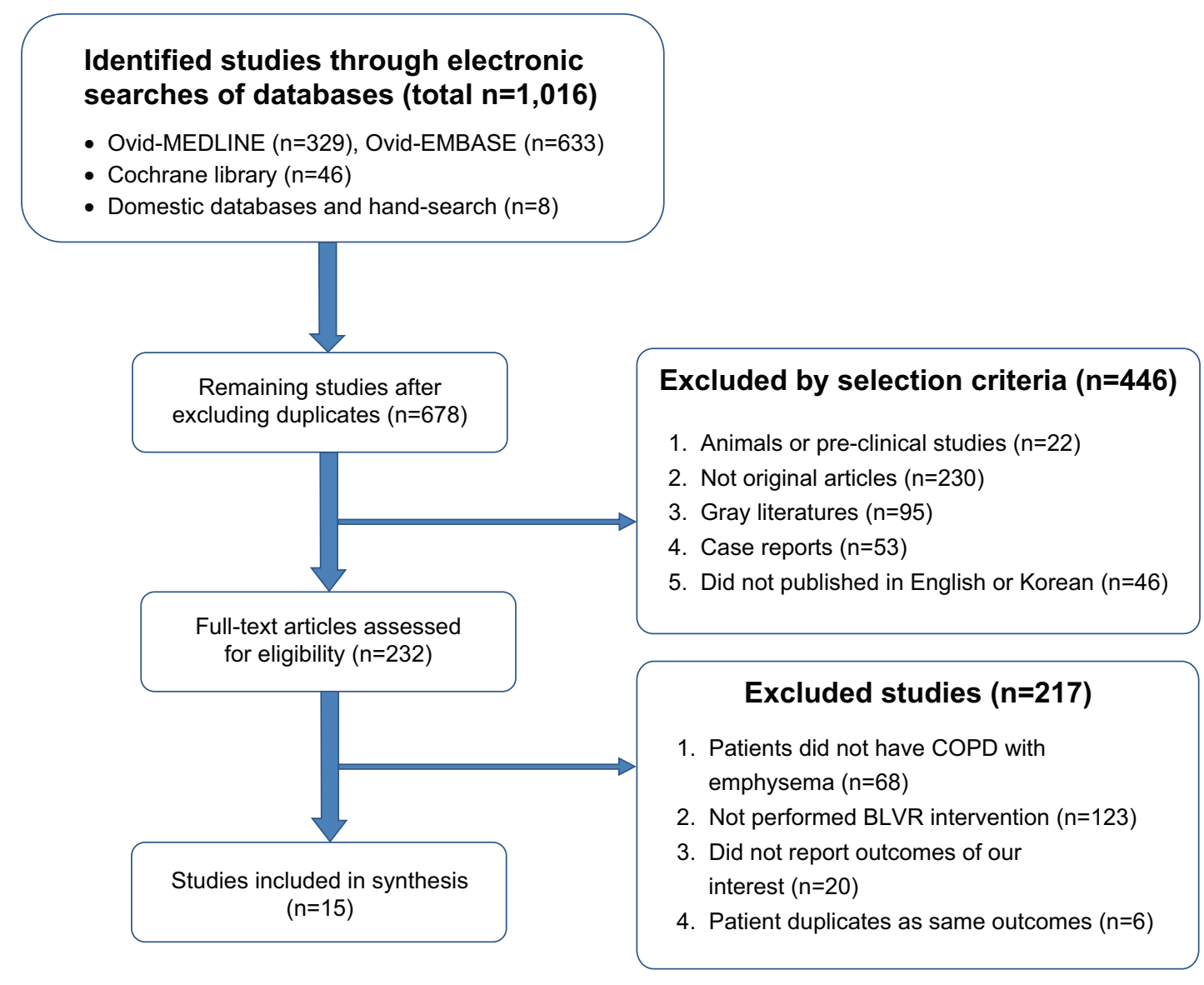

Figure I Flow diagram for identification of selected articles.

Abbreviation: BLVR, bronchoscopic lung volume reduction.

\section{Clinical effectiveness}

Two RCTs included in the meta-analysis compared the intervention group with the control group that performed only medical treatment. Each outcome measure, such as $\mathrm{FEV}_{1}, 6 \mathrm{MWD}$, cycle workload, and SGRQ, was reported (Figure 2). Six months after, the mean change from baseline and standard error were calculated for each group. The MDs with $95 \%$ CI were compared between the intervention group and the control group. The $\mathrm{FEV}_{1}$ improved in the intervention group compared with the control group ( $\mathrm{MD}=6.71$ ). 6MWD and cycle workload in the intervention group also increased (MD=15.66, $\mathrm{MD}=4.43$ ). The SGRQ score in the intervention group decreased compared with the control group ( $\mathrm{MD}=-4.29)$.

Although not included in the meta-analysis, the other $\mathrm{RCT}^{12}$ reported that for BLVR compared with the sham procedure (bronchoscopy) over 3 months, computed tomography (CT) lung volume and number of SGRQ responders were increased in the intervention group by $24.2 \%$ (8/33) compared with the control group (0/33). However, $\mathrm{FEV}_{1}$ and $6 \mathrm{MWD}$ were no different between the two groups.

One retrospective cohort study ${ }^{13}$ performed quantitative CT image analysis before and after the procedure. After 6 months of observation of lung volume, target lobe volume in the intervention group decreased from baseline $(-0.451 \mathrm{cc}$, $P<0.0001)$, but not in the control group $(-0.0051 \mathrm{cc}$, $P=0.70)$.

\section{Clinical effectiveness according to fissure completeness}

The subgroup was evaluated for the presence of complete fissure, and several outcomes were compared. The results are shown in Table 2. For patients with complete fissure, the $\mathrm{FEV}_{1}$ change from baseline in the BLVR group was higher than in the control group for both 6 months (MD=15.29, $P<0.001$ ) and 12 months ( $\mathrm{MD}=17.65, P<0.001)$. However, for the patients with incomplete fissure, mean change of $\mathrm{FEV}_{1}$ and 6MWD was not different. 


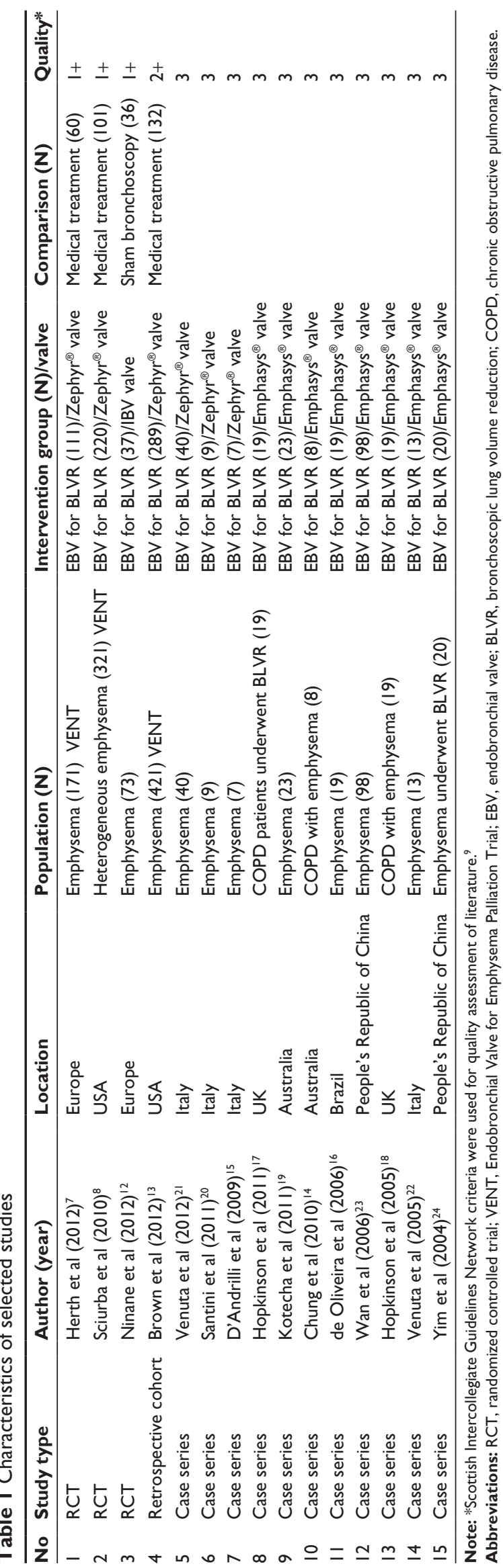

In addition, one case series study reported that 5-year survival rate of the fissure group was $83.3 \%$, whereas the nonfissure group was $24.0 \%$ by log-rank test $(P=0.0036) .{ }^{21}$

\section{Safety-related outcomes}

Three RCTs reported safety: ${ }^{7,8,12}$ two of them ${ }^{7,8}$ compared BLVR with medical treatment, and the other $\mathrm{RCT}^{12}$ compared BLVR with sham bronchoscopy. Firstly, one of the $\mathrm{RCTs}^{7}$ compared BLVR with medical treatment and found that mortality over 12 months occurred in $5.4 \%$ of the BLVR group of 111 participants and in $6.7 \%$ of the control group of 60 participants, although it was not statistically different. An independent committee of the RCT judged that the death and the BLVR procedure were not related. Respiratory failure occurred only in the intervention group (9.0\%). However, after 12 months, the occurrence of respiratory failure was not different between the two groups. The incidence of pneumothorax after the procedure occurred only in the intervention group ( $8.2 \%$ ), yet the incidence of pneumothorax was not statistically different. Massive hemoptysis occurred only in the intervention group ( $0.9 \%)$.

In the other RCT study, ${ }^{8}$ death occurred only in the intervention group (2.7\%) of 220 participants, although there was no significant difference between the two groups $(P=0.19)$. Causes of death were three cases of respiratory failure not associated with BLVR, one ischemic colitis, one cancer, and one massive hemoptysis. Respiratory failure occurred only in the intervention group (1.4\%). Pneumothorax occurred only in the BLVR group (4.1\%). One massive hemoptysis occurred in the intervention group. There was no statistically significant difference between the BLVR group and the control group.

One RCT ${ }^{12}$ compared BLVR with bronchoscopy as a sham procedure. During the 3-month follow-up period, adverse events were classified according to severity grade: serious, severe, moderate, or mild. Serious adverse events were seen in the intervention group in $18.9 \%$ of 37 participants and in $11.1 \%$ of the control group of 36 participants. The incidence rate was not different between the two groups $(P=0.214)$.

Because the follow-up period of RCT studies was less than 12 months, ten case series studies were selected for safety review. The follow-up observation period ranged from 1 month up to 5 years. Three of the studies ${ }^{19,21,23}$ reported death, but it was not related to the procedure. Of the major complications, seven pneumothorax cases were reported, and the incidence rate ranged from $2.5 \%(2 / 40)$ to $23 \%(3 / 13)$. Pneumonia distal to valve was $5.2 \%(1 / 19)$ in one study. ${ }^{18}$ 


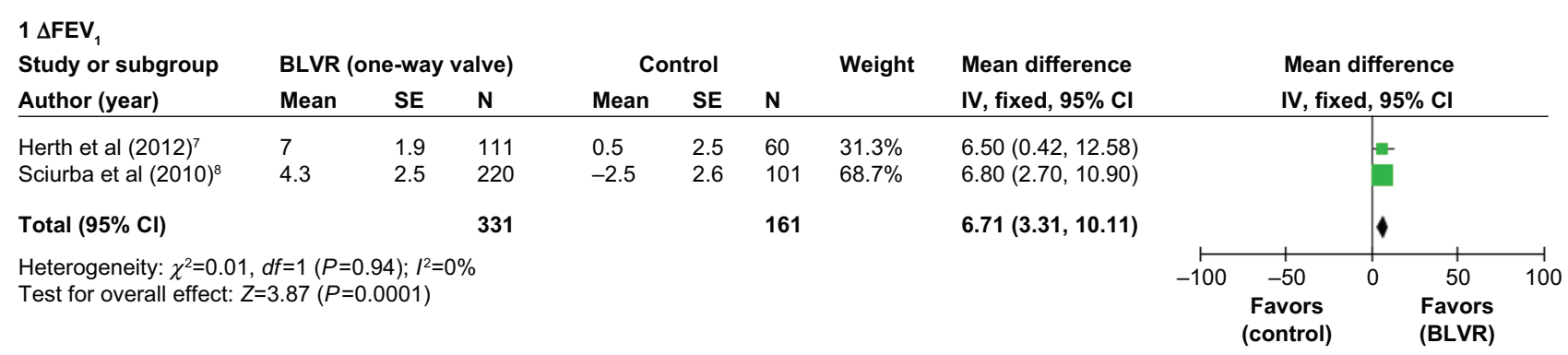

\section{$2 \triangle 6 M W D$}

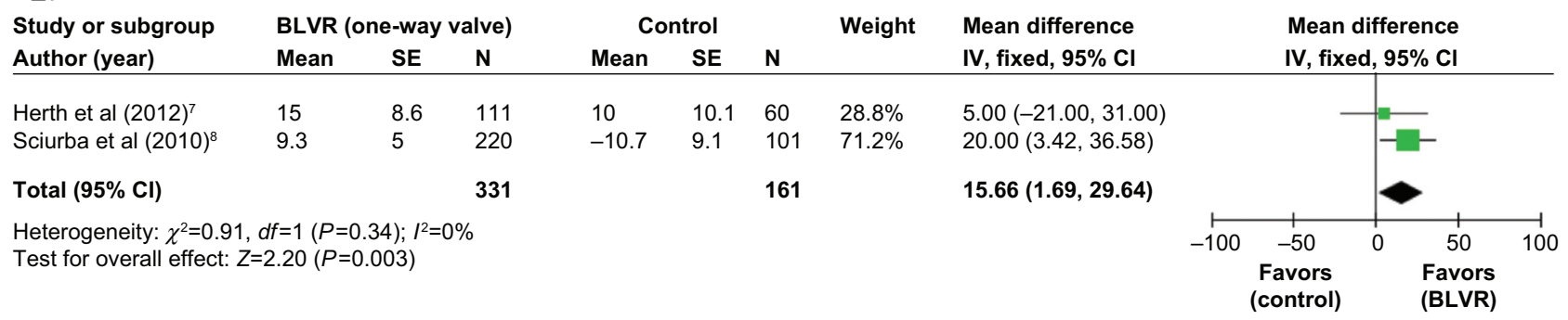

\section{$3 \Delta$ Cycle workload}

Study or subgroup

Author (year)

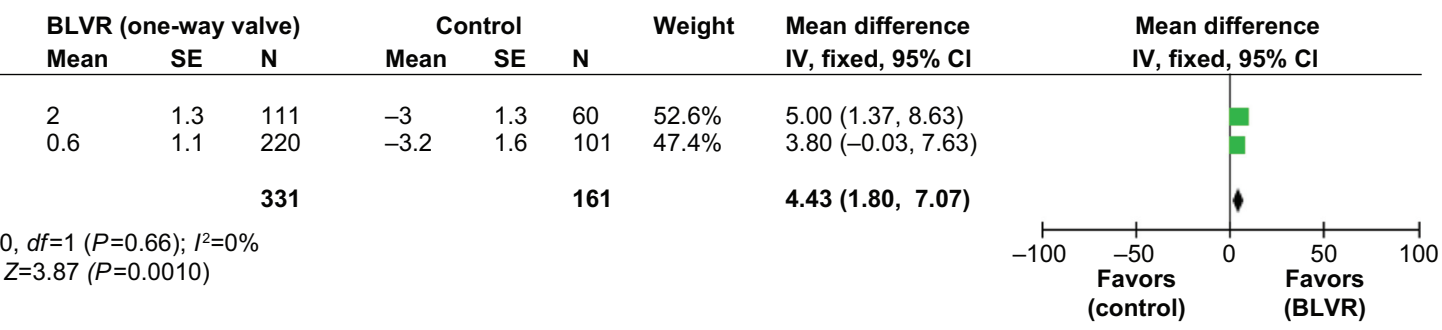

\section{$4 \triangle$ SGRQ score}

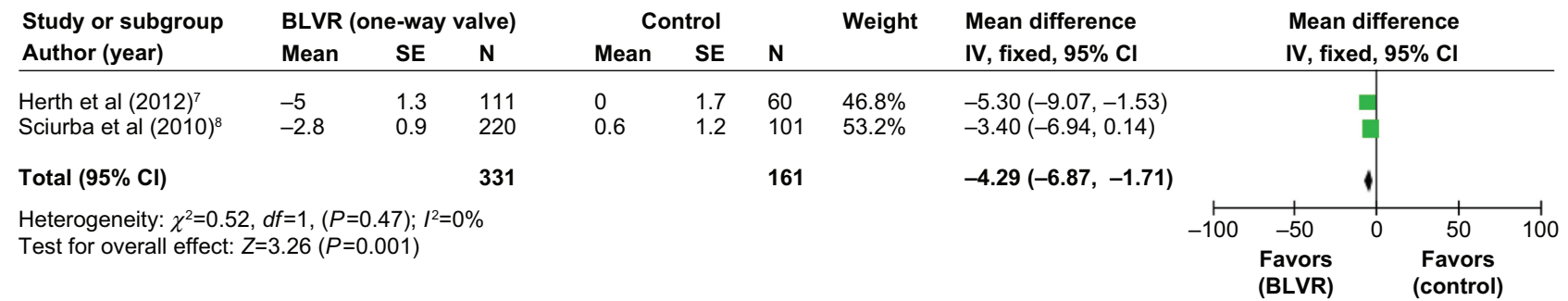

Figure 2 Forest plots of effectiveness outcomes - mean change differences from baseline between BLVR group and control group.

Abbreviations: $\mathrm{BLVR}$, bronchoscopic lung volume reduction; $\triangle \mathrm{FEV}_{1}$, change in forced expiratory volume in I second; SE, standard error; IV, independent variable; $\mathrm{Cl}$, confidence interval; $\triangle 6 \mathrm{MWD}$, change in 6-minute walking distance; $\triangle$ Cycle workload, change in cycle workload; $\Delta \mathrm{SGRQ}$, change in St George's Respiratory Questionnaire.

\section{Discussion}

BLVR using a one-way valve is a procedure for COPD patients with severe emphysema. Theoretically, a one-way valve inserted into the bronchus helps air outflow from the target lobe during expiration, and blocks air inflow into the lobe during inspiration. This mechanism improves the overall lung function and relieves the symptom of dyspnea by reducing the lung volume of the target lobe. ${ }^{5}$ However, even after the procedure, there are often collapse failures by collateral ventilation, in which case the effect of the procedure is reduced. The known appropriate population for the BLVR procedure is heterogeneous emphysema patients, and currently two types of bronchial valve are used. One is the duckbill-type valve; the other is the umbrella valve. ${ }^{25}$ Differences in the structure of these valves may impact on efficacy and safety, so only the duckbill-type valve was considered in this study.

The UK and European health technology assessment reports, based on a synthesis of the literature, did not include RCT studies and focused on case studies. For evaluation of safety and efficacy related to BLVR, these assessments recommended more RCTs. ${ }^{6}$ In a large RCT study, the Endobronchial Valve for Emphysema Palliation Trial (VENT) was included in the published results. VENT's selection criteria were similar to those for surgical lung volume reduction. ${ }^{26}$ Only 6 months of data were 


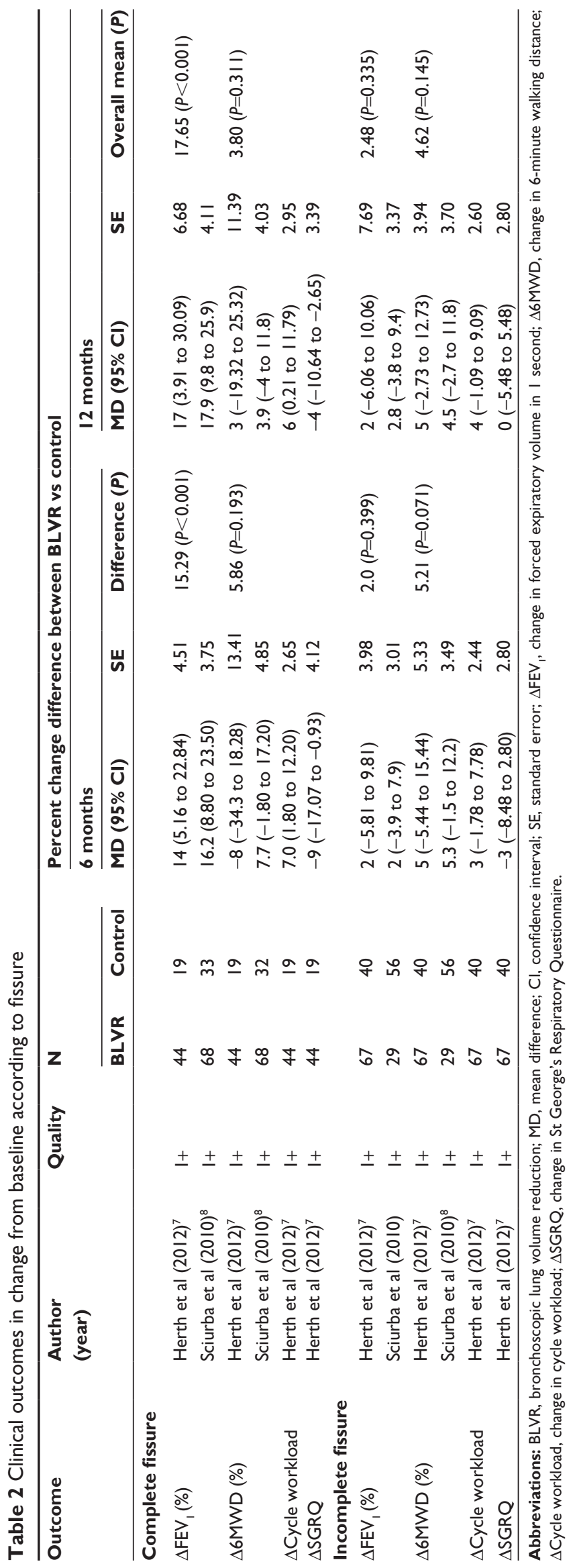

available for quantitative synthesis for two of the RCTs included in the meta-analysis, so long-term effect could not be analyzed. One of the RCT studies compared BLVR to sham bronchoscopy, so it was not included in this metaanalysis. ${ }^{12}$ This was because both the comparator with VENT and the time period were different. ${ }^{12}$ Based on the result of meta-analysis, BLVR is effective in lung function, such as $\mathrm{FEV}_{1}, 6 \mathrm{MWD}$, and cycle workload changes, compared with the group who maintained medical treatment. In addition, SGRQ score significantly decreased in the intervention group compared with the control group. One study's findings based on the modified Medical Research Council reported significant improvement $(P=0.04)$ between the intervention and sham groups. Conversely, during 3-month follow-up of other RCTs, compared with a sham procedure, both $\mathrm{FEV}_{1}$ and 6MWD were not different. These results suggest that the follow-up period after the procedure may influence its effectiveness. ${ }^{12}$ In one $\mathrm{RCT}^{12}$ and one cohort study, ${ }^{13}$ in chest CT scans, lung volume increased significantly in the intervention group. One case series ${ }^{21}$ followed 40 patients' $\mathrm{FEV}_{1}$ and found improvement for up to 5 years.

A previous study of collateral circulation between lobes reported that minimizing interlobar collaterals is the important key for success of BLVR intervention. ${ }^{27}$ As identified in VENT, the intact fissure (CT phase $90 \%$ intact) is related to a successful BLVR procedure. When complete fissure between lobes had been confirmed on CT, there was less collateral ventilation. This factor then affected the success of a treatment BLVR. Identifying this factor can be important to target group selection. ${ }^{28}$ In this study, the meta-analysis result shows that the lung function improvement was significant in the complete fissure group as opposed to the incomplete fissure group. Also in the observational case series, studies reported that atelectasis, heterogeneity, and lobe completeness can affect BLVR success. ${ }^{17,28}$ These results suggest that appropriate patient selection and bronchial valve position identification are an important area of research, and that an evidence-based approach is needed.

In a safety review, $\mathrm{VENT}^{7,8}$ reported deaths in the follow-up period of 12 months, but there was no evidence directly relating these deaths to the intervention group. The incidence rate was not different between intervention group and control group. Complication rates such as respiratory failure and pneumothorax incidence rate were higher compared with the control group, but most complications relieved after valve removal or palliative treatment. Although only having a short follow-up period of 3 months, for the RCT compared with bronchoscopy, the incidence rate 
of complications was not different between the intervention group and the control group. ${ }^{12}$ Most patients involved in these studies had severe emphysema and were considered a high-risk population for receiving surgery. Since BLVR is a less invasive procedure than surgery, safety could be determined as acceptable.

There were no studies that directly compared the effect of the BLVR procedure on lung volume reduction as a means to examine its safety. However, when considering the severity of the patient and ethical issues, direct comparison was possible. It can be recommended that indirect comparison or long-term observational studies are used to determine safety and effectiveness.

\section{Conclusion}

Our results suggest that BLVR may be an effective and safe procedure for the treatment of severe COPD patients with emphysema, based on existing studies. Furthermore, the intervention showed lung function improvement in patients with complete fissure, whereas patients with incomplete fissure showed no response. Therefore, more studies are needed to further investigate target patient selection and patient safety.

\section{Acknowledgment}

This study was funded by the National Evidence-Based Healthcare Collaborating Agency (NECA), Project No HTA-2013-025.

\section{Disclosure}

The authors have no conflicts of interest to disclose.

\section{References}

1. The Korean Academy of Tuberculosis and Respiratory Disease. COPD Clinical Practice Guideline. 2012.

2. Mannino DM, Buist AS. Global burden of COPD: risk factors, prevalence, and future trends. Lancet. 2007;370(9589):765-773.

3. Global Initiative for Chronic Obstructive Lung Disease. Global strategy for the diagnosis, management, and prevention of chronic obstructive pulmonary disease [web page on the Internet]. 2014. Available from: http://www.goldcopd.org/guidelines-global-strategy-for-diagnosismanagement.html. Accessed January 29, 2015.

4. Tiong LU, Davies R, Gibson PG, et al. Lung volume reduction surgery for diffuse emphysema. Cochrane Database Syst Rev. 2006;18(4): CD001001.

5. Oh YM. Bronchoscopic lung volume reduction. Tuberc Respir Dis. 2006; 61(6):521-525.

6. Belgian Health Care Knowledge Centre. Endobronchial Valves in the Treatment of Severe Pulmonary Emphysema. A Rapid Health Technology Assessment. 2009. Available from: http://kce.fgov.be/sites/default/files/ page_documents/d20091027339.pdf. Accessed January 29, 2015.

7. Herth FJ, Noppen M, Valipour A, et al. Efficacy predictors of lung volume reduction with Zephyr valves in a European cohort. Eur Respir J. 2012;39(6):1334-1342.
8. Sciurba FC, Ernst A, Herth FJ, et al. A randomized study of endobronchial valves for advanced emphysema. $N$ Engl J Med. 2010;363(13): 1233-1244.

9. Scottish Intercollegiate Guidelines Network. Critical appraisal: notes and checklists [web page on the Internet]. Available from: http:// www.sign.ac.uk/methodology/checklists.html. Accessed January 29, 2015.

10. Higgins JP, Green S. Cochrane Handbook for Systematic Reviews of Interventions. Vol 5: Wiley Online Library; 2008.

11. Kim S, Park J, Lee H, et al. NECA's guidance for undertaking systematic reviews and meta-analyses for intervention. National Evidence-based Healthcare Collaborating Agency; 2011.

12. Ninane V, Geltner C, Bezzi M, et al. Multicentre European study for the treatment of advanced emphysema with bronchial valves. Eur Respir J. 2012;39(6):1319-1325.

13. Brown MS, Kim HJ, Abtin FG, et al. Emphysema lung lobe volume reduction: effects on the ipsilateral and contralateral lobes. Eur Radiol. 2012;22(7):1547-1555.

14. Chung SC, Peters MJ, Chen S, Emmett L, Ing AJ. Effect of unilateral endobronchial valve insertion on pulmonary ventilation and perfusion: a pilot study. Respirology. 2010;15(7):1079-1083.

15. D'Andrilli A, Vismara L, Rolla M, et al. Computed tomography with volume rendering for the evaluation of parenchymal hyperinflation after bronchoscopic lung volume reduction. Eur J Cardiothorac Surg. 2009; 35(3):403-407.

16. de Oliveira HG, Macedo-Neto AV, John AB, et al. Transbronchoscopic pulmonary emphysema treatment: 1 -month to 24-month endoscopic follow-up. Chest. 2006;130(1):190-199.

17. Hopkinson NS, Kemp SV, Toma TP, et al. Atelectasis and survival after bronchoscopic lung volume reduction for COPD. Eur Respir J. 2011;37(6):1346-1351.

18. Hopkinson NS, Toma TP, Hansell DM, et al. Effect of bronchoscopic lung volume reduction on dynamic hyperinflation and exercise in emphysema. Am J Respir Crit Care Med. 2005;171(5):453-460.

19. Kotecha S, Westall GP, Holsworth L, Pham A, Williams TJ, Snell GI. Long-term outcomes from bronchoscopic lung volume reduction using a bronchial prosthesis. Respirology. 2011;16(1):167-173.

20. Santini M, Fiorelli A, Vicidomini G, Di CV, Messina G, Laperuta P. Endobronchial treatment of giant emphysematous bullae with one-way valves: a new approach for surgically unfit patients. Eur J Cardiothorac Surg. 2011;40(6):1425-1431.

21. Venuta F, Anile M, Diso D, et al. Long-term follow-up after bronchoscopic lung volume reduction in patients with emphysema. Eur Respir J. 2012;39(5):1084-1089.

22. Venuta F, de GT, Rendina EA, et al. Bronchoscopic lung-volume reduction with one-way valves in patients with heterogenous emphysema. Ann Thorac Surg. 2005;79(2):411-416.

23. Wan IY, Toma TP, Geddes DM, et al. Bronchoscopic lung volume reduction for end-stage emphysema: report on the first 98 patients. Chest. 2006; 129(3):518-526.

24. Yim AP, Hwong TM, Lee TW, et al. Early results of endoscopic lung volume reduction for emphysema. J Thorac Cardiovasc Surg. 2004; 127(6):1564-1573.

25. Ernst A, Anantham D. Bronchoscopic lung volume reduction. Pulm Med. 2011;2011:610802.

26. Strange C, Herth FJ, Kovitz KL, et al. Design of the Endobronchial Valve for Emphysema Palliation Trial (VENT): a non-surgical method of lung volume reduction. BMC Pulm Med. 2007;7:10.

27. Salanitri J, Kalff V, Kelly M, Holsworth L, Williams T, Snell G. 133 Xenon ventilation scintigraphy applied to bronchoscopic lung volume reduction techniques for emphysema: relevance of interlobar collaterals. Intern Med J. 2005;35(2):97-103.

28. Shah PL, Herth FJ. Current status of bronchoscopic lung volume reduction with endobronchial valves. Thorax. 2014;69(3):280-286. 


\section{Publish your work in this journal}

The International Journal of COPD is an international, peer-reviewed journal of therapeutics and pharmacology focusing on concise rapid reporting of clinical studies and reviews in COPD. Special focus is given to the pathophysiological processes underlying the disease, intervention programs, patient focused education, and self management protocols.

This journal is indexed on PubMed Central, MedLine and CAS. The manuscript management system is completely online and includes a very quick and fair peer-review system, which is all easy to use. Visit http://www.dovepress.com/testimonials.php to read real quotes from published authors.

Submit your manuscript here: http://www.dovepress.com/international-journal-of-chronic-obstructive-pulmonary-disease-journal 